\title{
Visualization of lattice dynamics and atomic motion in WSe2 monolayer
}

\section{Tony E. Karam}

Tony E. Karam, "Visualization of lattice dynamics and atomic motion in WSe2 monolayer," Proc. SPIE 10907, Synthesis and Photonics of Nanoscale Materials XVI, 109070D (4 March 2019); doi: 10.1117/12.2510389 


\title{
Visualization of lattice dynamics and atomic motion in $\mathrm{WSe}_{2}$ monolayer
}

\author{
Tony E. Karam* \\ Biology Center for Ultrafast Science and Technology - Arthur Amos Noyes Laboratory of \\ Chemical Physics - California Institute of Technology - Pasadena, CA 91125, United States
}

\begin{abstract}
Atomically-thin materials have drawn great interest due to their exotic properties leading to a variety of promising new applications. However, the out-of-equilibrium dynamics of these materials are still not fully understood. Ultrafast electron diffraction (UED) is a powerful technique for the investigation of the transient structural dynamics of monolayers with unprecedented spatiotemporal resolution. The UED results of $\mathrm{WSe}_{2}$ monolayers are reported and the suppression of the intensity of the Bragg diffraction spots following laser excitation is attributed to electron-phonon and phonon-phonon scattering. A two-temperature model is used to accurately describe these dynamics due to the low thermal conduction of the monolayer. Furthermore, the reported lifetimes are fit using a single-mode relaxation time approximation.
\end{abstract}

\section{*TKaram@edmundoptics.com}

\section{Introduction}

Atomically-thin materials have been widely investigated in the last decade soon after the first exfoliation of graphene due to their numerous potential applications. In particular, 2D transition metal dichalcogenides (TMDCs) possess bandgaps on the order of 1.6-2.1 eV, leading to unique optoelectronic, mechanical, chemical, and thermal properties. ${ }^{1-5} \mathrm{~A}$ direct to indirect bandgap shift is observed in $\mathrm{MX}_{2}$ compounds ( $\mathrm{M}=\mathrm{Mo}, \mathrm{W}$ and $\left.\mathrm{X}=\mathrm{S}, \mathrm{Se}\right)$ due to the shift of the valence band hills and conduction band valleys in the Brillouin zone. ${ }^{6-9}$ Edge dislocation in single layer transition metal disulfides $\mathrm{MS}_{2}(\mathrm{M}=\mathrm{Mo}$ or $\mathrm{W})$ leading to local distortions of the crystal lattice was previously predicted $^{10}$, which can have remarkable effects on the photoluminescence and electrical conductivity of the material. ${ }^{11}$ 
Ultrafast electron diffraction (UED) ${ }^{12-16}$ provides a unique tool for the study of transient structural dynamics in monolayers and atomically-thin samples due to the large scattering cross section of electrons as compared to X-rays. ${ }^{17-19}$ In this technique, an optical pump pulse initiating the dynamics in the sample, is followed by electron pulses that probe the structural changes at various pump-probe time delays. Using UED, processes such as correlated atomic motions, bond dilation, and structural transformation in nanostructures can be investigated on the order of the nuclear motion by combining the high temporal resolution of ultrafast lasers and the atomic-scale spatial resolution of electrons. ${ }^{20-22}$

In this proceeding, the ultrafast structural dynamics of monolayer $\mathrm{WSe}_{2}$ are reported. The suppression of the intensity of the Bragg diffraction spots is attributed to the displacement of the atoms following laser excitation and can be characterized by electron-phonon and phononphonon scattering. Two-temperature model is used to accurately describe the out-of-equilibrium dynamics due to the low thermal conduction of the monolayer. Similarly to graphene, the lifetimes observed from the UED experiment can be described by a single-mode relaxation time approximation.

\section{Experimental Section}

The $\mathrm{WSe}_{2}$ monolayers are CVD grown on sapphire substrates then transferred on TEM grids. The Raman spectrum (Figure 1 (a)) reveals a peak centered at $250 \mathrm{~cm}^{-1}$ followed by a smaller shoulder centered at $260 \mathrm{~cm}^{-1}$. These peaks are attributed to the $E_{2 g}^{1}$ and $A_{1 g}$ phonon modes, respectively. ${ }^{23}$ The photoluminescence spectrum (Figure 1(b)) shows a strong emission band centered at $790 \mathrm{~nm}$ which is indicative of the indirect to direct bandgap transition in the monolayer. ${ }^{24}$ This emission band originates from $\mathrm{K} \rightarrow \Gamma$ transition where the conduction band minimum is located at the $\mathrm{K}$ point. ${ }^{25}$. An atomic force microscopy (AFM) image of the $\mathrm{WSe}_{2}$ monolayer flake deposited on sapphire is shown in Figure 1 (c). The AFM measurements (Figure 1 (d)) reveal a monolayer thickness of around $0.652 \mathrm{~nm}$, corresponding to the height of a single unit cell. The $\mathrm{WSe}_{2}$ samples deposited on TEM grids are annealed in a tube furnace at 160 for 
2 hours at a pressure of $12 \mathrm{mTorr}$ right before they are loaded in the ultra-high vacuum chamber for the ultrafast electron diffraction experiments.

The UED setup ${ }^{18}$ consists of a Ti:sapphire laser generating $800 \mathrm{~nm}$ pulses with a $100 \mathrm{fs}$ duration and a repetition rate of $2 \mathrm{kHz}$. The fundamental beam is split into pump and probe arms using a beam splitter. The pump pulses are P-polarized using a polarizing beam cube and are then focused into the sample contained in a UHV chamber. The probe beam pass through a frequency tripler to generate $266 \mathrm{~nm}$ UV pulses that are subsequently focused into a $\mathrm{LaB}_{6}$ photocathode. The generated photoelectrons are then accelerated to $30 \mathrm{keV}$ and tightly focused to a spatial overlap with pump beam at the sample surface. To minimize space-charge effects, the number of electrons in each pulse is maintained at around 300 photoelectrons. The spatial overlap between pump and probe beams is ensured by maximizing the transmission of both beams through a $150-\mu \mathrm{m}$ aperture at the sample plane. The sample is mounted on a computercontrolled five-axis stage and the pump-probe temporal delay is generated using a delay stage. The time-dependent diffraction patterns are recorded using a microchannel plate/phosphor screen coupled to a CCD working in the gate mode.

\section{Results and Discussion}

Figure 2 (a) shows a representative electron diffraction pattern of $\mathrm{WSe}_{2}$ monolayer showing distinctive Bragg diffraction spots. The diffraction intensity spectrum obtained by azimuthally averaging the diffraction pattern and its curve fitting is shown in Figure 2 (b). A one-dimensional diffraction curve is obtained as a function of the scattering vector. The timedependent changes in the diffraction pattern are then recovered by fitting the location and area of the diffraction peaks at various pump-probe time delays using a piecewise-linear background and a Lorentzian function. The time-resolved intensity change of the Bragg diffraction spots are shown in Figure 3 (a) at 5.0, 5.9, 7.1, and $8.2 \mathrm{~mJ} / \mathrm{cm}^{2}$ laser excitation fluences. These temporal profiles are obtained by averaging over the six first-order diffraction spots shown in Figure 2 (a).

The room temperature thermal conductivity of layered $\mathrm{WSe}_{2}$ monolayer is known to be extremely low due to localized lattice vibrations leading to its uniform heating with negligible thermal conduction using a laser pulse where the spot size of the photo-excitation is much larger 
than the flake. ${ }^{26}$ Hence, a two-temperature model can be employed to accurately model the suppression of the Bragg diffraction intensity and calculate the rise in temperature of the sample following $100 \mathrm{fs}$ full-width at half maximum (FWHM) incident laser pulse. The two-coupled nonlinear differential equations are given by:

$$
\begin{gathered}
C_{e} \frac{\partial}{\partial t} T_{e}=\frac{\partial}{\partial z}\left(k_{e} \frac{\partial}{\partial z} T_{e}\right)-g\left(T_{e}-T_{l}\right)+S(z, t) \\
C_{l} \frac{\partial}{\partial t} T_{l}=g\left(T_{e}-T_{l}\right)
\end{gathered}
$$

where $e$ and $l$ subscripts denote electron and lattice, respectively. $C$ and $k$ are the heat capacities and the thermal conductivity, respectively. $S(z, t)$ is the time- and space-dependent heating term introduced by the femtosecond laser pulse and $g$ is the electron-phonon coupling constant. The values of $k_{e}, C_{l}$, and $C_{e}$ used are provided in Refs. 27, 28, and 29. ${ }^{27-29}$ The best fit twotemperature model results are obtained for $g=1.5 \times 10^{6} \mathrm{~W} \cdot \mathrm{m}^{-2} \cdot \mathrm{K}^{-1}$. In contrast, the electronphonon coupling constant of graphene/ $\mathrm{WSe}_{2}$ heterostructure is $0.5 \times 10^{6} \mathrm{~W} \cdot \mathrm{m}^{-2} \cdot \mathrm{K}^{-1} \cdot{ }^{30} \mathrm{The}$ value of the lattice temperature $T_{l}$ obtained from the simulation is used to calculate the change in intensity of the Bragg diffraction spots using the Debye-Waller model given by: ${ }^{31}$

$$
\frac{I(t)}{I_{0}}=\exp \left[2 s_{h k l}^{2}\left(\frac{<u^{2}\left(T_{0}\right)>-<u^{2}\left(T_{l}\right)>}{4}\right)\right]
$$

$u^{2}(T)$ is the atomic mean square displacement given by:

$$
<u^{2}(T) \geq \frac{3 \hbar^{2}}{2 m k_{b} \theta_{D}}\left[1+4\left(\frac{T}{\theta_{D}}\right)^{2} \int_{0}^{\frac{\theta_{D}}{T}} \frac{x}{\exp (x)-1} \cdot d x\right.
$$

where $m$ is the effective mass of the unit cell, $k_{b}$ is the Boltzmann's constant, and $\theta_{D}$ is the Debye temperature. As shown in Figure 3 (a), the two-temperature model fit (dashed lines) shows good agreement with the experimental results at different fluences.

Figure 3 (b) shows a plot of the lifetimes obtained from the single-exponential fit of the transient intensity change versus laser excitation fluence, along with corresponding peak lattice temperatures. These lifetimes can be described using a single relaxation time approximation of the Boltzmann transport equation (BTE) that neglects phonon reabsorption due to their low 
probability of occurrence. ${ }^{32-34}$ The average relaxation lifetime of the phonon modes in $2 \mathrm{D}$ materials is given by ${ }^{16}$ :

$$
\tau \propto \frac{1}{1+\alpha T}
$$

where $\alpha=\frac{2 K_{b}}{\hbar \omega}, K_{b}$ is Boltzmann constant, and $\hbar \omega$ is LO-phonon energy. The calculated lifetime values shown in Figure 3 (b) are calculated for $\hbar \omega=31.25 \mathrm{meV}$, which corresponds to the LO-phonon energy in monolayer $\mathrm{WSe}_{2}{ }^{35,36}$ The calculated results are in good agreement with the experimental lifetime values at the various corresponding temperatures.

\section{Conclusion}

This work highlights UED as a powerful tool for the investigation of the transient state structural dynamics of atomically-thin materials with unprecedented spatiotemporal resolution. The time-dependent suppression of the intensity of the Bragg diffraction spots is reported and the dynamics are attributed to electron-phonon and phonon-phonon scattering. Two temperature model is used to accurately describe these dynamics due to the negligible thermal conduction of the monolayer. Furthermore, the experimental lifetimes of the $\mathrm{WSe}_{2}$ monolayer exhibit similar behavior as observed in graphene where the experimental lifetimes obtained at various excitation fluences are accurately described by a single-mode relaxation time approximation.

\section{ACKNOWLEDGMENT}

Generous financial support for this work was provided by the Gordon and Betty Moore Foundation. This manuscript is dedicated to the memory of our mentor Professor Ahmed H. Zewail. 


\section{Figures}
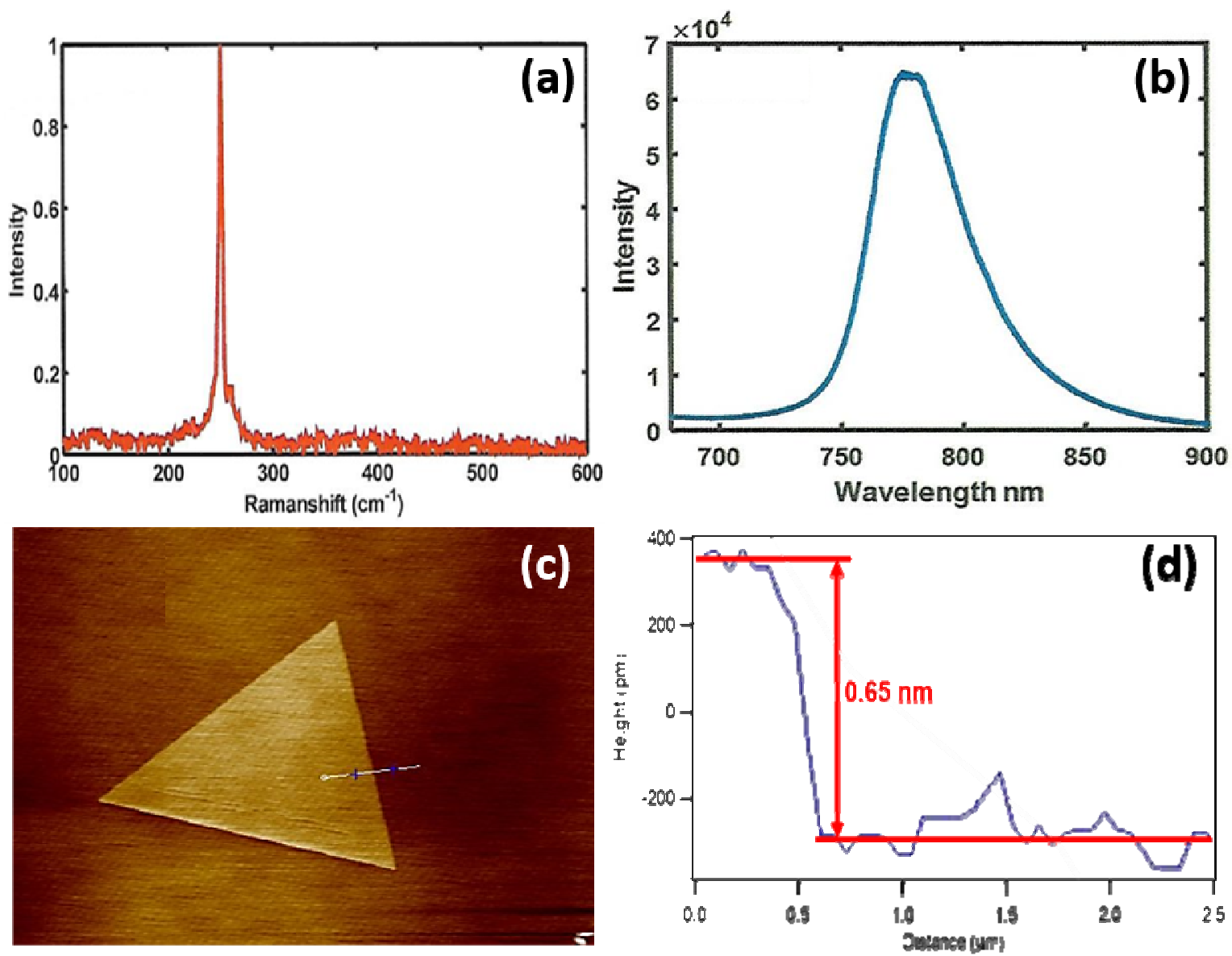

Figure 1. (a) Raman spectrum of a $\mathrm{WSe}_{2}$ monolayer on a sapphire substrate. (b) Photoluminescence spectrum of a $\mathrm{WSe}_{2}$ monolayer on a sapphire substrate following $532 \mathrm{~nm}$ laser excitation. (c) AFM image of a $\mathrm{WSe}_{2}$ monolayer flake mounted on a sapphire substrate. (d) The height of the sample is $0.65 \mathrm{~nm}$, corresponding to the thickness of a single unit cell. 

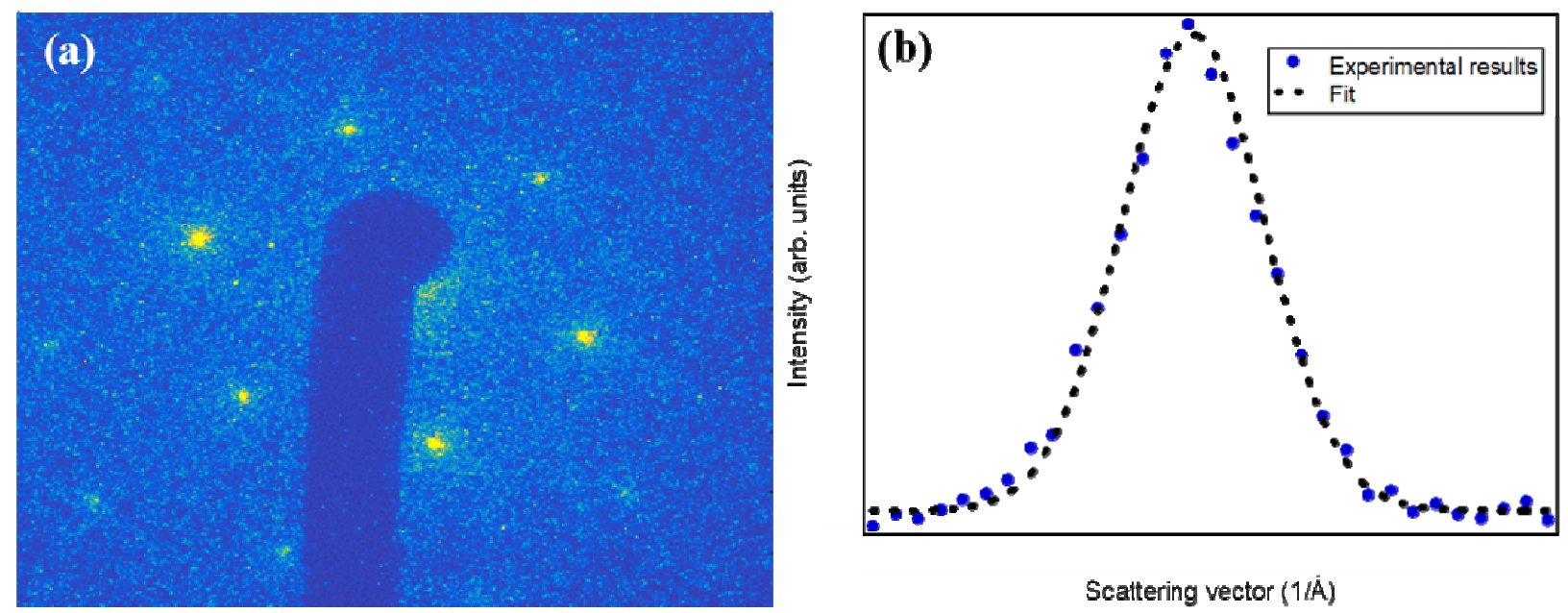

Figure 2. (a) Electron diffraction image of the $\mathrm{WSe}_{2}$ monolayer. (b) Diffraction intensity spectrum obtained by azimuthally averaging the diffraction pattern, and its curve fitting.
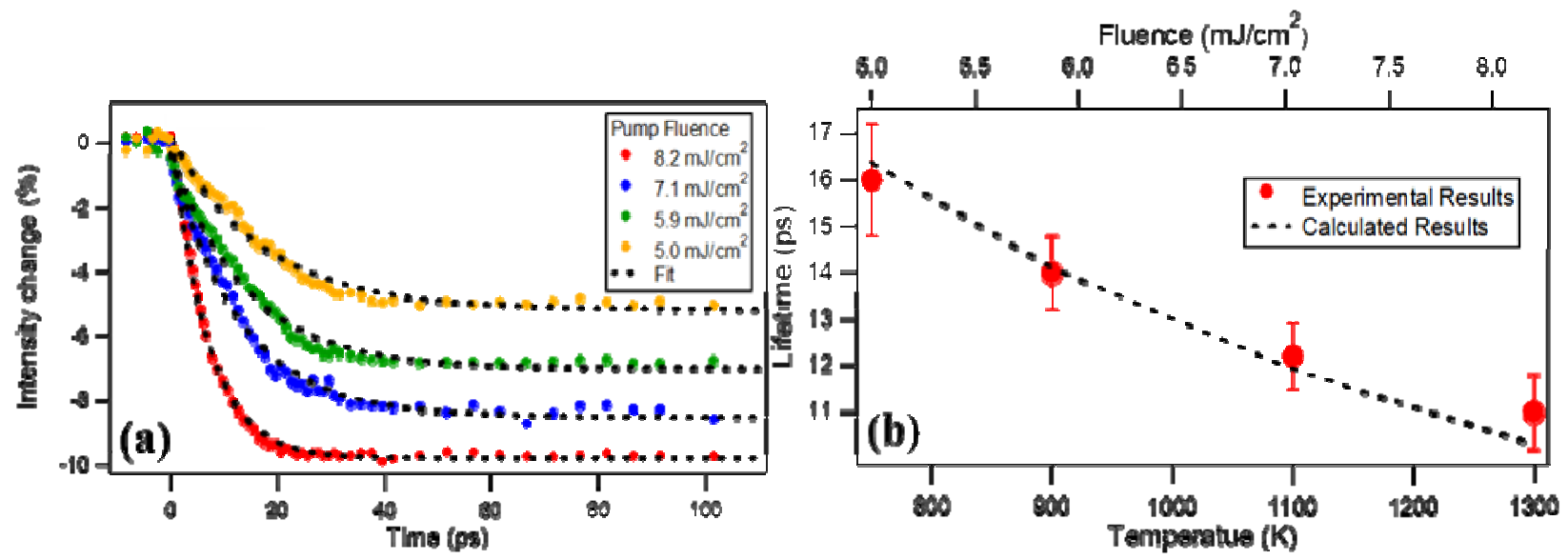

Figure 3. (a) Time-resolved diffraction spot intensity change at various laser excitation fluences. The dynamics are fit using a two-temperature model. (b) Plot of the lifetimes obtained from the single-exponential fit of the transient intensity change versus laser excitation fluence, and the corresponding temperatures. 


\section{References}

(1) Novoselov, K., Jiang, D., Schedin, F., Booth, T., Khotkevich, V., Morozov, S., Geim, A., "Two-dimensional atomic crystals," Proc. Nat. Acad. Sci. 102, 10451-10453 (2005).

(2) Novoselov, K. S., Geim, A. K., Morozov, S. V., Jiang, D., Zhang, Y., Dubonos, S. V., Grigorieva, I. V., Firsov, A. A., "Electric field effect in atomically thin carbon films," Science 306, 666-669 (2004).

(3) Yang, L., Majumdar, K., Liu, H., Du, Y., Wu, H., Hatzistergos, M., Hung, P.,

Tieckelmann, R., Tsai, W., Hobbs, C., "Chloride molecular doping technique on 2D materials: $\mathrm{WS}_{2}$ and $\mathrm{MoS}_{2}$," Nano Lett. 14, 6275-6280 (2014).

(4) Wang, Q. H., Kalantar-Zadeh, K., Kis, A., Coleman, J. N., Strano, M. S., "Electronics and optoelectronics of two-dimensional transition metal dichalcogenides," Nat. Nanotech. 7, 699-712 (2012).

(5) Osada, M., Sasaki, T., "Two dimensional dielectric nanosheets: novel nanoelectronics from nanocrystal building blocks," Adv. Mater. 24, 210-228 (2012).

(6) Mak, K. F., Lee, C., Hone, J., Shan, J., Heinz, T. F., “Atomically thin $\mathrm{MoS}_{2}$ : a new direct-gap semiconductor,” Phys. Rev. Lett. 105, 136805 (2010).

(7) Splendiani, A., Sun, L., Zhang, Y., Li, T., Kim, J., Chim, C.-Y., Galli, G., Wang, F., "Emerging photoluminescence in monolayer $\mathrm{MoS}_{2}$," Nano Lett. 10, 1271-1275 (2010).

(8) Zhao, W., Ghorannevis, Z., Chu, L., Toh, M., Kloc, C., Tan, P.-H., Eda, G., "Evolution of electronic structure in atomically thin sheets of $\mathrm{WS}_{2}$ and $\mathrm{WSe}_{2}$, , ACS Nano 7, 791-797 (2012).

(9) Tongay, S., Zhou, J., Ataca, C., Lo, K., Matthews, T. S., Li, J., Grossman, J. C., Wu, J., "Thermally driven crossover from indirect toward direct bandgap in 2D semiconductors: $\mathrm{MoSe}_{2}$ versus $\mathrm{MoS}_{2}$," Nano Lett. 12, 5576-5580 (2012).

(10) Zou, X., Liu, Y., Yakobson, B. I., "Predicting dislocations and grain boundaries in twodimensional metal-disulfides from the first principles," Nano Lett. 13, 253-258 (2012).

(11) Van Der Zande, A. M., Huang, P. Y., Chenet, D. A., Berkelbach, T. C., You, Y., Lee, G.H., Heinz, T. F., Reichman, D. R., Muller, D. A., Hone, J. C., "Grains and grain boundaries in highly crystalline monolayer molybdenum disulphide." Nat. Mater. 12, 554-561 (2013).

(12) Baum, P., Zewail, A. H., "Breaking resolution limits in ultrafast electron diffraction and microscopy," Proc. Nat. Acad. Sci. 103, 16105-16110 (2006).

(13) Morrison, V. R., Chatelain, R. P., Tiwari, K. L., Hendaoui, A., Bruhács, A., Chaker, M., Siwick, B. J., "A photoinduced metal-like phase of monoclinic $\mathrm{VO}_{2}$ revealed by ultrafast electron diffraction," Science 346, 445-448 (2014).

(14) Miller, R. D., "Femtosecond crystallography with ultrabright electrons and x-rays: Capturing chemistry in action," Science 343, 1108-1116 (2014).

(15) Mannebach, E. M., Li, R., Duerloo, K.-A., Nyby, C., Zalden, P., Vecchione, T., Ernst, F., Reid, A. H., Chase, T., Shen, X., "Dynamic structural response and deformations of monolayer $\mathrm{MoS}_{2}$ visualized by femtosecond electron diffraction," Nano Lett. 15, 6889-6895 (2015).

(16) $\mathrm{Hu}$, J., Vanacore, G. M., Cepellotti, A., Marzari, N., Zewail, A. H., "Rippling ultrafast dynamics of suspended 2D monolayers, graphene," Proc. Nat. Acad. Sci. 201613818 (2016). 
(17) Mannebach, E. M., Li, R., Duerloo, K.-A., Nyby, C., Zalden, P., Vecchione, T., Ernst, F., Reid, A. H., Chase, T., Shen, X., "Dynamic structural response and deformations of monolayer MoS2 visualized by femtosecond electron diffraction," Nano Lett. 15, 6889-6895 (2015).

(18) Hu, J., Vanacore, G. M., Cepellotti, A., Marzari, N., Zewail, A. H., "Rippling ultrafast dynamics of suspended 2D monolayers, graphene," Proc. Natl. Acad. Sci. U. S. A. 113, 13818 (2016).

(19) Zewail, A. H., "4D ultrafast electron diffraction, crystallography, and microscopy," Annu. Rev. Phys. Chem. 57, 65-103 (2006).

(20) Kealhofer, C., Schneider, W., Ehberger, D., Ryabov, A., Krausz, F., Baum, P., “Alloptical control and metrology of electron pulses," Science 352, 429-433 (2016).

(21) Gao, M., Lu, C., Jean-Ruel, H., Liu, L. C., Marx, A., Onda, K., Koshihara, S.-y., Nakano, Y., Shao, X., Hiramatsu, T., "Mapping molecular motions leading to charge delocalization with ultrabright electrons," Nature 496, 343 (2013).

(22) Hassan, M. T., Baskin, J., Liao, B., Zewail, A., "High-temporal-resolution electron microscopy for imaging ultrafast electron dynamics.," Nat. Photon. 11, 425 (2017).

(23) Tongay, S., Suh, J., Ataca, C., Fan, W., Luce, A., Kang, J. S., Liu, J., Ko, C., Raghunathanan, R., Zhou, J., "Defects activated photoluminescence in two-dimensional semiconductors: interplay between bound, charged, and free excitons," Sci. Rep. 3, 2657 (2013).

(24) Horzum, S., Sahin, H., Cahangirov, S., Cudazzo, P., Rubio, A., Serin, T., Peeters, F., "Phonon softening and direct to indirect band gap crossover in strained single-layer $\mathrm{MoSe}_{2}$," Phys. Rev. B 87, 125415 (2013).

(25) Zhao, W., Ribeiro, R. M., Toh, M., Carvalho, A., Kloc, C., Castro Neto, A., Eda, G., "Origin of indirect optical transitions in few-layer $\mathrm{MoS}_{2}, \mathrm{WS}_{2}$, and $\mathrm{WSe}_{2}$," Nano Lett. 13, 56275634 (2013).

(26) Chiritescu, C., Cahill, D. G., Nguyen, N., Johnson, D., Bodapati, A., Keblinski, P., Zschack, P., "Ultralow thermal conductivity in disordered, layered WSe ${ }_{2}$ crystals," Science 315, 351-353 (2007).

(27) Zhou, W.-X., Chen, K.-Q., "First-principles determination of ultralow thermal conductivity of monolayer $\mathrm{WSe}_{2}$," Scientific reports 5 (2015).

(28) Kim, J.-Y., Choi, S.-M., Seo, W.-S., Cho, W.-S., "Thermal and electronic properties of exfoliated metal chalcogenides," Bulletin of the Korean Chemical Society 31, 3225-3227 (2010). (29) Callanan, J. E., Hope, G., Weir, R. D., Westrum, E. F., "Thermodynamic properties of tungsten ditelluride $\left(\mathrm{WTe}_{2}\right) \mathrm{I}$. The preparation and lowtemperature heat capacity at temperatures from $6 \mathrm{~K}$ to $326 \mathrm{~K}$," The Journal of Chemical Thermodynamics 24, 627-638 (1992).

(30) Massicotte, M., Schmidt, P., Vialla, F., Watanabe, K., Taniguchi, T., Tielrooij, K.-J., Koppens, F. H., "Photo-thermionic effect in vertical graphene heterostructures," Nature communications 7 (2016).

(31) Schäfer, S., Liang, W., Zewail, A. H., "Primary structural dynamics in graphite," New Journal of Physics 13, 063030 (2011).

(32) Fugallo, G., Lazzeri, M., Paulatto, L., Mauri, F., “Ab initio variational approach for evaluating lattice thermal conductivity,” Phys. Rev. B 88, 045430 (2013).

(33) Xu, G., Broholm, C., Reich, D. H., Adams, M., "Triplet waves in a quantum spin liquid," Phys. Rev. Lett. 84, 4465 (2000). 
(34) Fugallo, G., Cepellotti, A., Paulatto, L., Lazzeri, M., Marzari, N., Mauri, F., "Thermal conductivity of graphene and graphite: collective excitations and mean free paths," Nano Lett. 14, 6109-6114 (2014).

(35) Tonndorf, P., Schmidt, R., Böttger, P., Zhang, X., Börner, J., Liebig, A., Albrecht, M., Kloc, C., Gordan, O., Zahn, D. R., "Photoluminescence emission and Raman response of monolayer $\mathrm{MoS}_{2}, \mathrm{MoSe}_{2}$, and WSe 2 ," Opt. Express 21, 4908-4916 (2013).

(36) Arora, A., Koperski, M., Nogajewski, K., Marcus, J., Faugeras, C., Potemski, M., "Excitonic resonances in thin films of $\mathrm{WSe}_{2}$ : from monolayer to bulk material," Nanoscale 7, 10421-10429 (2015). 